\title{
Trees and Reflection Groups
}

\author{
Humberto Luiz Talpo* \\ Instituto de Matemática, IMECC \\ Universidade Estadual de Campinas, Campinas-SP, Brasil. \\ humberto@ime.unicamp.br \\ Marcelo Firer \\ Instituto de Matemática, IMECC \\ Universidade Estadual de Campinas, Campinas-SP, Brasil. \\ mfirer@ime.unicamp.br
}

Submitted: Mar 19, 2003; Accepted: Mar 5, 2005; Published: Mar 14, 2005

Mathematics Subject Classifications: 20E08, 05C05

\begin{abstract}
We define a reflection in a tree as an involutive automorphism whose set of fixed points is a geodesic and prove that, for the case of a homogeneous tree of degree $4 k$, the topological closure of the group generated by reflections has index 2 in the group of automorphisms of the tree.
\end{abstract}

\section{Basic Concepts}

Although many of the constructions in this work make sense in the wide context of trees, and with minor modifications even to graphs or $\Lambda$-trees, we are concerned with homogeneous trees, so that the definitions are introduced in this restricted context. All the concepts and definitions needed may be found in either [1] or [4]. We start considering the free monoid $M(X)$ over a non-empty alphabet $X$ with $N \in \mathbb{N}$ elements. Here $\mathbb{N}$ denotes the set of non-negative integers, an assumption that will be useful for the notation to be established in section 2. The elements of $X$ are called letters of the alphabet. The product is just the concatenation and the empty word $\emptyset$ plays the role of the identity element. Given a word $x=x_{i_{1}} x_{i_{2}} \ldots x_{i_{k}}$, we denote its length by $|x|=k$. A prefix of the word $x=x_{i_{1}} x_{i_{2}} \ldots x_{i_{k}}$ is a sub-word $x=x_{i_{1}} x_{i_{2}} \ldots x_{i_{l}}$, with $l \leq k$. This induces a partial order on $M(X)$ :

$$
x \leq y \text { if and only if } x \text { is a prefix of } y \text {. }
$$

*Author supported by FAPESP, proc. no. 99/09839-4 
The tree $\Gamma:=\Gamma(M)$ is just the Cayley graph of the monoid $M$. The set of vertices is identified with $M$ and a vertex $x$ is connected to a vertex $x x_{i}$, where $x_{i} \in X$ is a letter. The distance in $\Gamma$ is defined by the length function:

$$
d_{\Gamma}(x, y):=|x|+|y|-2|z|
$$

where $z$ is the maximal common prefix of $x$ and $y$. With this distance, we find that two vertices $x$ and $y$ are connected by an edge if and only if $d_{\Gamma}(x, y)=1$, and in this case, we say they are adjacent to each other. A path of length $n$ in $\Gamma$ connecting vertices $x$ and $y$ is a map

$$
\alpha:\{0,1,2, \ldots, n\} \longmapsto \Gamma
$$

such that $\alpha(0)=x, \alpha(n)=y$ and $d_{\Gamma}(\alpha(i), \alpha(i+1))=1$, for every $i \in\{0,1, \ldots, n-1\}$. With this definition we find that the distance between two vertices is the length of the shortest path connecting them.

We denote respectively by $S(x, R)$ and $B(x, R)$ the usual metric sphere and closed ball of $\Gamma$, centered at $x$ with radius $R$. In particular, $S(x, 1)$ is the set of vertices adjacent to $x$. An isometry of the tree is a bijection (one-to-one and onto mapping) of the set of vertices of $\Gamma$, preserving the distance. An isometry certainly preserves adjacency, that is, vertices with distance 1. Conversely, a bijection of the set of vertices that preserves adjacency preserves distances in $\Gamma$. Thus an automorphism of the graph $\Gamma$ (i.e., a bijection preserving adjacency) is an isometry of $\Gamma$ and vice versa. We let Aut $(\Gamma)$ denote the group of automorphisms (isometries) of $\Gamma$.

Every vertex of $\Gamma(M)$ is adjacent to exactly $N+1$ other vertices, excepts for the distinguished vertex defined by the empty word.

If we consider two copies $\Gamma^{\prime}$ and $\Gamma^{\prime \prime}$ of the tree $\Gamma(M)$ and add a single edge, connecting the vertex of $\Gamma^{\prime}$ labelled by $\emptyset^{\prime}$ to the vertex of $\Gamma^{\prime \prime}$ labelled by $\emptyset^{\prime \prime}$, we eliminate the distinguished role of the empty word and get a homogeneous tree $\Gamma$, that is, a tree where the number $\operatorname{Degree}_{\Gamma}(x)$ of vertices adjacent to $x$ is constant. We extend the distances on $\Gamma^{\prime}$ and $\Gamma^{\prime \prime}$ to a distance on $\Gamma$ by defining

$$
d(x, y)=|x|_{\Gamma^{\prime}}+|y|_{\Gamma^{\prime \prime}}+1,
$$

whenever $x \in \Gamma^{\prime}$ and $y \in \Gamma^{\prime \prime}$. All other concepts, such as path and adjacency, isometry, spheres and balls, are extended in a similar manner. Since we are assuming that Degree $_{\Gamma}(x)$ is constant, we denote it by Degree $(\Gamma)$ and call it the degree of $\Gamma$. Homogeneous trees with even degree, the kind we should focus at, arise naturally as the Cayley graph of finitely generated free groups.

An integer interval is a subset of $\mathbb{Z}$ of one of the kinds $\mathbb{Z}, \mathbb{N}$ or $\mathrm{I}_{a, b}:=\{n \in \mathbb{Z} \mid a \leq n \leq b\}$, with $a, b \in \mathbb{Z}$. A subset $\gamma(\mathrm{I}) \subset \Gamma$ is called a geodesic, $a$ geodesic ray or a geodesic segment if $\gamma: \mathrm{I} \rightarrow \Gamma$ is a map defined on an integer interval respectively of type $\mathbb{Z}, \mathbb{N}$ or $\mathrm{I}_{a, b}$ such that $d(\gamma(n), \gamma(m))=|n-m|$ for every $n, m \in \mathrm{I}$. We call the map $\gamma: \mathrm{I} \rightarrow \Gamma$ a parametrization but often make no distinction between the map $\gamma$ and its image $\gamma(\mathrm{I})$. We denote by $[x, y]$ the geodesic segment joining the vertices $x, y \in \Gamma$. 


\section{Reflections on Trees}

There are many possibilities to define a reflection on a tree. The minimal condition for a map $\phi: \Gamma \rightarrow \Gamma$ to resemble what is commonly known as a reflection in geometry, is to demand $\phi$ to be an involutive automorphism. Indeed, this is the definition adopted by Moran in [2]. We can get a good feeling of how much this condition is minimal from the fact that it implies that every automorphism of a homogeneous tree is the product of at most two reflections. In [2, Theorem 4.13], it is established for the group of automorphisms of arbitrary tree fixing a given vertex $x_{0}$. When the tree $\Gamma$ is a homogeneous this holds in the full group of tree-automorphisms Aut $(\Gamma)$ (Gadi Moran, private communication). In this work, we adopt a much more restrictive definition:

Definition 1 A reflection on a tree $\Gamma$ is a automorphism $\phi: \Gamma \longrightarrow \Gamma$, satisfying:

1. $\phi$ is a involution, i.e., $\phi^{2}=\mathrm{Id}$.

2. The set of fixed points of $\phi$ is a geodesic $\gamma \subset \Gamma$, i.e., there is a geodesic $\gamma$ such that $\phi(x)=x \Leftrightarrow x \in \gamma$.

Under these conditions, we say that $\phi$ is a reflection in the geodesic $\gamma$.

From here on, we assume that $\Gamma$ is a homogeneous tree. With this condition, the choice of a particular geodesic as fixed points of a reflection is irrelevant, as will be shown in Proposition 4. We start with some definitions:

Definition 2 If $X$ is a subset of a given group, we denote by $\langle X\rangle$ the subgroup generated by $X$. Given a geodesic $\gamma$ in a tree $\Gamma, \mathcal{R}_{\gamma}$ is the set of all reflections in $\gamma$ and thus $\left\langle\mathcal{R}_{\gamma}\right\rangle$ is the subgroup of Aut $(\Gamma)$ generated by $\mathcal{R}_{\gamma}$.

The abundance of automorphisms of a homogeneous tree is well known (see e.g [2, p. 253]). We will reproduce here a consequence we need in the sequel, introducing through its proof the labelling of vertices to be used later.

Lemma 3 Given geodesics $\gamma$ and $\beta$ in a homogeneous tree $\Gamma$, there is a $\psi \in$ Aut $(\Gamma)$ such that $\psi(\gamma)=\beta$.

Proof. Let us assume for the moment that $\gamma \cap \beta$ is infinite and $\gamma \neq \beta$. In this case, this intersection is a geodesic ray and we loose no generality by assuming that $\gamma(n)=\beta(n)$ if and only if $n \leq 0$. We denote $\gamma(0)=\beta(0)$ by $x_{0}$ and label the other vertices of $\Gamma$ starting from this vertex. If $N=\operatorname{Degree}(\Gamma)$, there are exactly $N$ vertices adjacent to $x_{0}$, and we label them as $x_{0,1}, x_{0,2}, \ldots, x_{0, N}$, assuming that $\gamma(1)=x_{0,1}$ and $\beta(1)=x_{0, N}$. Since $\Gamma$ is homogeneous, each $x_{0, i}$ is adjacent to exactly $N$ vertices, $N-1$ of them at distance 2 from $x_{0}$. We label them as $x_{0, i, 1}, x_{0, i, 2}, \ldots, x_{0, i, N-1}$, assuming that $\gamma(2)=x_{0,1,1}$ and $\beta(2)=x_{0, N, 1}$. Proceeding in this way, each vertex $x \in \Gamma$ with $d\left(x_{0}, x\right)=k$ is labelled as $x_{0, i_{1}, i_{2}, \ldots, i_{k}}$, with $i_{1} \in\{1,2, \ldots, N\}$ and $i_{j} \in\{1,2, \ldots, N-1\}$ if $j \geq 2$, and $x_{0,1,1, \ldots, 1}=\gamma(k), x_{0, N, 1, \ldots, 1}=\beta(k)$. 
Note that the distance between two vertices can be easily determined from their labels. Let $x, y \in \Gamma$ be vertices labelled as $x=x_{0, i_{1}, i_{2}, \ldots, i_{k}}$ and $y=x_{0, j_{1}, j_{2}, \ldots, j_{l}}$ and define

$$
r=\max \left\{0 \leq s \leq \min \{k, l\} \mid i_{t}=j_{t} \text { if } t \leq s\right\}
$$

where, by definition, $i_{0}=j_{0}=0$. Then, we find that

$$
d(x, y)=k+l-2 r .
$$

We denote by $\gamma^{+}$and $\beta^{+}$the geodesic rays $\gamma(\mathbb{N})$ and $\beta(\mathbb{N})$ respectively. Because of the choices made in the labelling, we find that the vertices of $\gamma^{+}$and $\beta^{+}$are labelled by the sequences

$$
\begin{aligned}
& \gamma^{+}=x_{0}, x_{0,1}, x_{0,1,1}, \ldots x_{0,1,1, \ldots, 1}, \ldots \\
& \beta^{+}=x_{0}, x_{0, N}, x_{0, N, 1}, \ldots x_{0, N, 1, \ldots, 1}, \ldots
\end{aligned}
$$

We define a map $\psi: \Gamma \rightarrow \Gamma$ by

$$
\psi(x)=\left\{\begin{array}{cc}
x_{0, N, i_{2}, \ldots, i_{k}} & \text { if } x=x_{0,1, i_{2}, \ldots, i_{k}} \\
x_{0,1, i_{2}, \ldots, i_{k}} & \text { if } x=x_{0, N, i_{2}, \ldots, i_{k}} \\
x & \text { otherwise }
\end{array} .\right.
$$

It follows from formula (1) that $\psi \in \operatorname{Aut}(\Gamma)$. Moreover, $\psi$ is an involution and by construction, $\psi\left(\gamma^{+}\right)=\beta^{+}$and $\left.\psi\right|_{\gamma \cap \beta}=\mathrm{Id}$, so that $\psi(\gamma)=\beta$.

Let us assume now that $\gamma \cap \beta$ is finite but not empty. In this case this intersection is a geodesic segment (possibly consisting of a unique vertex) and we loose no generality by assuming $\gamma(n)=\beta(n)$ if and only if $a \leq n \leq 0$, for some $a \leq 0$. We label the vertices of $\Gamma$, starting from $x_{0}:=\gamma(0)=\beta(0)$, in the same way we did before, assuming again that the vertices of $\gamma^{+}:=\gamma(\mathbb{N})$ are labelled by $x_{0}, x_{0,1}, x_{0,1,1}, \ldots, x_{0,1,1, \ldots, 1}, \ldots$ and the vertices of $\beta^{+}:=\beta(\mathbb{N})$ by $x_{0}, x_{0, N}, x_{0, N, 1}, \ldots, x_{0, N, 1, \ldots, 1}, \ldots$ The map $\psi$ defined as in $(2)$, is again an automorphism and $\psi\left(\gamma^{+}\right)=\beta^{+}$. But $\psi(\gamma) \cap \beta$ is the geodesic ray $\beta(\mathrm{I}), \mathrm{I}=\{n \in \mathbb{Z} \mid n \geq a\}$ so we are in the situation of the first case.

At last, we consider the case when $\gamma \cap \beta=\emptyset$. Let $\alpha_{1}$ be the (unique) geodesic segment joining $\gamma$ to $\beta$ with endpoints $x_{0}$ and $y_{0}$ in $\gamma$ and $\beta$ respectively. We write $\gamma=\gamma^{+} \cup \gamma^{-}$ and $\beta=\beta^{+} \cup \beta^{-}$with $\gamma^{+} \cap \gamma^{-}=x_{0}$ and $\beta^{+} \cap \beta^{-}=y_{0}$. Then, $\alpha:=\gamma^{+} \cup \alpha_{1} \cup \beta^{+}$is a geodesic intersecting $\beta$ in the ray $\beta^{+}$. Applying the first case, we find an automorphism $\psi_{1}$ such that $\psi_{1}(\alpha)=\beta$. But this implies that $\psi_{1}\left(\gamma^{+}\right) \subset \beta$, and so $\psi_{1}(\gamma) \cap \beta$ is a geodesic ray. Again, we find an automorphism $\psi_{2}$ such that $\psi_{2} \circ \psi_{1}(\gamma)=\beta$.

We note that both $\psi_{1}$ and $\psi_{2}$ are involutions and actually we proved that any geodesic is mappable onto any other geodesic by a product of two involutions.

Proposition 4 Given geodesics $\gamma$ and $\beta$ in a homogeneous tree $\Gamma, \mathcal{R}_{\beta}$ and $\mathcal{R}_{\gamma}$ are conjugated in Aut $(\Gamma)$.

Proof. This follows immediately from Lemma 3 and the fact that conjugacy carries a geodesic to a geodesic.

Corollary 5 Given geodesics $\gamma$ and $\beta$ in a homogeneous tree $\Gamma$, the subgroups $\left\langle\mathcal{R}_{\gamma}\right\rangle$ and $\left\langle\mathcal{R}_{\beta}\right\rangle$ are conjugated in Aut $(\Gamma)$.

Proof. Follows immediately from proposition 4. 


\section{The Group Generated by Reflections}

The main question we address here is when an automorphism of $\Gamma$ may be described as a product of reflections. The following proposition asserts that not every automorphism may be produced by reflections. We start defining the displacement function of an automorphism $\varphi$ as $d_{\varphi}(x):=d(x, \varphi(x))$.

Proposition 6 Let $\phi_{1}, \phi_{2}, \ldots, \phi_{n}$ be reflections in a tree $\Gamma$ and $\varphi=\phi_{1} \circ \phi_{2} \circ \ldots \circ \phi_{n}$. Then $d_{\varphi}(x) \equiv 0 \bmod 2$ for every $x \in \Gamma$.

Proof. Given a reflection $\phi$ in the geodesic $\gamma$ and a vertex $x \in \Gamma$, the (unique) vertex $x_{0} \in \gamma$ such that $d\left(x_{0}, x\right)=d(x, \gamma)$ is the middle point of the geodesic segment $[x, \phi(x)]$ joining $x$ to $\phi(x)$. Since $d\left(x, x_{0}\right)=d\left(\phi(x), x_{0}\right)$ and $d(x, \phi(x))=d\left(x, x_{0}\right)+d\left(x_{0}, \phi(x)\right)$, we find that $d_{\phi}(x)=2 d\left(x, x_{0}\right) \equiv 0 \bmod 2$. Since the set of automorphisms with even displacement is a subgroup, it follows that $d_{\varphi}(x) \equiv 0 \bmod 2$, for $\varphi=\phi_{1} \circ \phi_{2} \circ \ldots \circ \phi_{n}$ a product of reflections.

This proposition says that automorphisms with displacement function assuming odd values can not be produced by reflections. The most we can expect is to produce the automorphisms which displacement function assumes only even values.

This is not a bad situation, since

$$
\operatorname{Aut}^{+}(\Gamma)=\left\{\varphi \in \operatorname{Aut}(\Gamma) \mid d_{\varphi}(x) \equiv 0 \bmod 2 \text { for every } x \in \Gamma\right\}
$$

is a subgroup of index 2 in Aut $(\Gamma)$ [3, Proposition 1]. We will prove (Theorem 15) that this expectation is not vain: the closure of the group generated by reflections is the subgroup $\operatorname{Aut}^{+}(\Gamma)$, if Degree $(\Gamma) \equiv 0 \bmod 4$.

It is known that given sets $Y \subseteq X$, and a subgroup $G \subseteq S_{X}$ (permutation group of $X)$ such that $\phi(Y)=Y$ for any $\phi \in G$, the restriction map

$$
\begin{aligned}
\Phi: G & \rightarrow H \subseteq S_{Y} \\
\phi & \mapsto \bar{\phi}:=\left.\phi\right|_{Y}
\end{aligned}
$$

is a group homomorphism, not necessarily onto. In the next proposition we will specify, in two circumstances, subgroups $G \subseteq S_{X}$ and $H \subseteq S_{Y}$ that turn the restriction map surjective. The first one is when we consider $G$ as Aut $(\Gamma)_{x_{0}}$, the stabilizer of $x_{0}$ in Aut $(\Gamma)$, and $H$ as Aut $(B)$, where $B:=B\left(x_{0}, R\right)$ is a metric ball. For stating the second circumstance, we define a diameter of $B$ as a maximal geodesic segment in $B$. Each diameter $[a, b]$ is such that $d(a, b)=2 R$ and $x_{0}$ is the middle point of $[a, b]$. We say that $\phi \in \operatorname{Aut}(B)$ is a reflection in $[a, b]$ if it is an involution that fixes exactly the vertices in $[a, b]$. We denote by $\mathcal{R}_{B,[a, b]}$ the set of reflections of $B$ in $[a, b]$ and thus $\left\langle\mathcal{R}_{B,[a, b]}\right\rangle$ denotes the subgroup of $\operatorname{Aut}(B)$ generated by $\mathcal{R}_{B,[a, b]}$. Let $\gamma$ be any geodesic containing the diameter $[a, b] \subset B$ and as usual, denote by $\left\langle\mathcal{R}_{\gamma}\right\rangle$ the subgroup of Aut $(\Gamma)$ generated by reflections in $\gamma$. In the next proposition we will prove that the restriction $\operatorname{map} \Phi: \operatorname{Aut}(\Gamma)_{x_{0}} \rightarrow \operatorname{Aut}(B)$ is surjective and maps $\mathcal{R}_{\gamma}$ onto $\mathcal{R}_{B,[a, b]}$. 
Proposition 7 Let $\Gamma$ be a homogeneous tree with Degree $(\Gamma)=4 k$. With the notation used above, let

$$
\begin{aligned}
\Phi: \operatorname{Aut}(\Gamma)_{x_{0}} & \rightarrow \operatorname{Aut}(B) \\
\phi & \mapsto \bar{\phi}:=\left.\phi\right|_{B}
\end{aligned}
$$

be the restriction map. Then,

1. $\Phi$ is surjective;

2. For any diameter $[a, b]$ of $B$ and any geodesic $\gamma$ containing $[a, b]$, the restriction map $\Phi$ maps $\mathcal{R}_{\gamma}$ onto $\mathcal{R}_{B,[a, b]}$.

\section{Proof.}

1. In the same manner we did in lemma 3, we can label the vertices of $\Gamma$, and hence of $B$, starting from the center $x_{0}$. By doing so, given vertices

$$
\begin{aligned}
& x=x_{0, i_{1}, i_{2}, \ldots, i_{l}} \\
& y=x_{0, j_{1}, j_{2}, \ldots, j_{k}}
\end{aligned}
$$

we have that $d\left(x_{0}, x\right)=l, d\left(x_{0}, y\right)=k$ and $d(x, y)=l+k-2 r$, where

$$
r=\max \left\{0 \leq s \leq \min \{k, l\} \mid i_{t}=j_{t} \text { if } t \leq s\right\}
$$

In other words, the distance between two vertices $x$ and $y$ depends only on its distance to the center $x_{0}$ and the length $r$ of its common prefix.

Given $\varphi \in \operatorname{Aut}(B)$, it must fix the center of the ball $x_{0}$ and it follows that $d\left(x_{0}, \varphi(x)\right)=d\left(x_{0}, x\right)$ for any $x \in B$. In other words, if

$$
x=x_{0, i_{1}, i_{2}, \ldots, i_{l}} \text { and } y=x_{0, j_{1}, j_{2}, \ldots, j_{k}}
$$

then

$$
\varphi(x)=x_{0, i_{1}^{\prime}, i_{2}^{\prime}, \ldots, i_{l}^{\prime}} \text { and } \varphi(y)=x_{0, j_{1}^{\prime}, j_{2}^{\prime}, \ldots, j_{k}^{\prime}}
$$

But

$$
d(x, y)=l+k-2 r
$$

and

$$
d(\varphi(x), \varphi(y))=l+k-2 r^{\prime}
$$

where $\quad r^{\prime}=\max \left\{0 \leq s \leq \min \{k, l\} \mid i_{t}^{\prime}=j_{t}^{\prime}\right.$ if $\left.t \leq s\right\}$, and since $d(x, y)=d(\varphi(x), \varphi(y))$ we find that $\min \left\{s \mid i_{s} \neq j_{s}\right\}=\min \left\{s \mid i_{s}^{\prime} \neq j_{s}^{\prime}\right\}$, that is, the maximal common prefix of $\varphi(x)$ and $\varphi(y)$ has the same length as the maximal common prefix of $x$ and $y$. In particular, the vertices $x$ and $y$ are adjacent (have distance 1) if and only if

$$
\begin{aligned}
& k=l+1 \text { and } y=x_{0, i_{1}, \ldots, i_{l}, j_{l+1}} \\
& \text { or } \\
& l=k+1 \text { and } x=x_{0, j_{1}, \ldots, j_{k}, i_{k+1}} .
\end{aligned}
$$


We want to show the existence of an automorphism $\phi \in \operatorname{Aut}(\Gamma)$ that extends $\varphi$. First of all we consider the projection $\pi: \Gamma \rightarrow B$ that associates to a vertex $x$ the closest vertex contained in $B$, that is, $\pi(x)$ is the last vertex in $B$ in the unique path from $x_{0}$ to $x$. Obviously $\pi(x)=x$ iff $x \in B$. We say that $\pi(x)$ is the root of $x$ in $B$.

If $x=x_{0, i_{1}, i_{2}, \ldots, i_{R}, \ldots, i_{R+l}}$ not in $B$ (hence $l \geq 1$ ) then $x_{0, i_{1}, i_{2}, \ldots, i_{R}}$, is its root in $B$. Its image under $\varphi$ must be in distance $R$ from $x_{0}$ and so it is labeled as $x_{0, i_{1}^{\prime}, i_{2}^{\prime}, \ldots, i_{R}^{\prime}}$. We define

$$
\phi\left(x_{0, i_{1}, i_{2}, \ldots, i_{R}, \ldots, i_{R+l}}\right):=x_{0, i_{1}^{\prime}, i_{2}^{\prime}, \ldots, i_{R}^{\prime}, i_{R+1} \ldots, i_{R+l}} .
$$

Thus, the restriction of $\phi$ to $B$ coincides with $\varphi$ and $\phi$ is one to one.

Let us show that $\phi \in \operatorname{Aut}(\Gamma)$. From what we noticed in $\left(^{*}\right)$, it is clear that $\phi$ preserves adjacency and is one to one, hence preserves distance. By construction, $\phi$ is surjective on $B$, so given a vertex $x=x_{0, i_{1}, i_{2}, \ldots, i_{R}, \ldots, i_{R+l}} \notin B$, there is $x^{\prime} \in B$ such that $\phi\left(x^{\prime}\right)=x_{0, i_{1}, i_{2}, \ldots, i_{R}}$. If $x^{\prime}=x_{0, i_{1}^{\prime}, i_{2}^{\prime}, \ldots, i_{R}^{\prime}}$ it follows $\phi\left(x_{0, i_{1}^{\prime}, i_{2}^{\prime}, \ldots, i_{R}^{\prime}, i_{R+1} \ldots, i_{R+l}}\right)=x$ and $\phi$ is surjective., that it follows that $\phi$ is indeed an automorphism of $\Gamma$ that extends $\varphi$.

2. For this part of the proposition, we will label the vertices starting from the center $x_{0}$ but with more specific conditions. Let $\gamma$ be a geodesic containing the diameter $[a, b]$ of $B:=B\left(x_{0}, R\right)$. We label the vertices of $\gamma$ adjacent to $x_{0}$ as $x_{0,1}$ and $x_{0,4 k}$. The following vertices of $\gamma$ are labeled as $x_{0,1,1, \ldots, 1}$ and $x_{0,4 k, 1,1, \ldots, 1}$, where the number of non-zero entries in the arrays $(0,1, \ldots, 1)$ and $(0,4 k, 1, \ldots, 1)$ is exactly the distance between the given vertex and $x_{0}$. So, we may assume that the diameter $[a, b]$ is labeled as

$$
a=x_{0,4 k, 1, \ldots, 1}, \ldots, x_{0,4 k, 1}, x_{0,4 k}, x_{0}, x_{0,1}, x_{0,1,1}, \ldots, \underset{\substack{0,1,1, \ldots, 1 \\ R \text { times }}}{ }=b .
$$

Given $\varphi \in \mathcal{R}_{B,[a, b]}$, none of the vertices adjacent to $x_{0}$ other then $x_{0,1}$ and $x_{0,4 k}$ is fixed by $\varphi$. So, if we label those vertices as $x_{0,2}, x_{0,3}, \ldots, x_{0,4 k-1}$, there is an involution $\sigma$ of the set $I:=\{2,3, \ldots, 4 k-1\}$ with no fixed point, such that $\varphi\left(x_{0, i}\right)=x_{0, \sigma(i)}$, whenever $i \in I$. Since $\varphi$ is an involution it follows that $\sigma$ is a product of $\frac{4 k-2}{2}=2 k-1$ disjoint transposition. So, we can relabel those vertices in such a manner that

$$
\sigma=(2,2 k+1)(3,2 k+2), \ldots,(2 k, 4 k-1) .
$$

We consider now the vertices in the branches starting at $x_{0, i}$ and $x_{0, \sigma(i)}$, for $i \in I$. Each vertex $x_{0, i, j}, i \in I$ is mapped by $\varphi$ to a vertex $x_{0, \sigma(i), j^{\prime}}$. So, we may assume that $j^{\prime}=j$, that is, we are making a label such that $\varphi\left(x_{0, i_{1}, i_{2}}\right)=x_{0, \sigma\left(i_{1}\right), i_{2}}$.

We observe that

$$
\begin{aligned}
\varphi\left(\varphi\left(x_{0, i_{1}, i_{2}}\right)\right) & =\varphi\left(x_{0, \sigma\left(i_{1}\right), i_{2}}\right) \\
& =x_{0, \sigma\left(\sigma\left(i_{1}\right)\right), i_{2}} \\
& =x_{0, i_{1}, i_{2}}
\end{aligned}
$$


so that this labeling is coherent with the fact that $\varphi^{2}=$ Id. As we did in the proof of the first part of the proposition, we consider the projection $\pi: \Gamma \rightarrow \gamma$ and say that $y \in \Gamma$ is the root of $x$ if $\pi(x)=y$.

Repeating this argument for the higher levels, we can label the elements of $B\left(x_{0}, R\right)$ not in $\gamma$ but whose root is $x_{0}$ in a way that for every $l \leq R$ and $i_{1} \in I$,

$$
\varphi\left(x_{0, i_{1}, i_{2}, \ldots, i_{l}}\right)=x_{0, \sigma\left(i_{1}\right), i_{2}, \ldots, i_{l}} .
$$

Exactly the same argument can be repeated for the elements in $B\left(x_{0}, R\right)$ whose roots are any point $x_{0,1,1, \ldots, 1}$ or $x_{0,4 k, 1, \ldots, 1}$ in the diameter $[a, b]$. To be more precise, every vertex $x \in B\left(x_{0}, R\right)$ other than $x_{0}$ is labelled as $x_{0, i_{1}, i_{2}, \ldots, i_{l}}$ with $i_{1} \in\{1,2, \ldots, 4 k\}$, $i_{2}, i_{3}, \ldots, i_{l} \in\{1,2, \ldots, 4 k-1\}$ and $l \in\{1,2, \ldots, R\}$. If $i_{1} \notin\{1,4 k\}$, then

$$
\varphi\left(x_{0, i_{1}, i_{2}, \ldots, i_{l}}\right)=x_{0, \sigma\left(i_{1}\right), i_{2}, \ldots, i_{l}}
$$

If $i_{1}=1$ or $i_{1}=4 k$ and $x \notin[a, b]$, we consider

$$
j_{0}=\min \left\{j=2,3, \ldots, l \mid i_{j} \neq 1\right\},
$$

and then

$$
\varphi\left(x_{0, i_{1}, 1, \ldots, 1, i_{j_{0}}, \ldots, i_{l}}\right)=x_{0, i_{1}, 1, \ldots, 1, \sigma\left(i_{j_{0}}\right), \ldots, i_{l}} .
$$

We remark that deleting the indices $i_{j_{0}}, \ldots, i_{l}$ we get the root of $x$ in the geodesic $\gamma$, so that $j_{0}=d\left(x_{0} \cdot \pi(x)\right)+1$.

Now, the vertices at distance more than $R$ from $x_{0}$ may be labeled with no restriction, just respecting the basic rule that vertices

$$
x_{i_{1}, i_{2}, i_{3}, \ldots, i_{r}} \text { and } x_{j_{1}, j_{2}, j_{3}, \ldots, j_{s}}
$$

are adjacent if and only if

$$
\begin{aligned}
& r=s+1 \text { and }\left(i_{1}, i_{2}, \ldots, i_{r-1}\right)=\left(j_{1}, j_{2}, \ldots j_{s}\right) \\
& \text { or } \\
& s=r+1 \text { and }\left(i_{1}, i_{2}, \ldots, i_{r}\right)=\left(j_{1}, j_{2}, \ldots j_{s-1}\right) .
\end{aligned}
$$

We define now a map $\phi: \Gamma \rightarrow \Gamma$ with the required property.

For $x \in \gamma$, let $\phi(x):=x$. Remember we labeled the vertices of the geodesic $\gamma$ as

$$
\ldots, x_{0,4 k, 1, \ldots, 1}, \ldots, x_{0,4 k, 1}, x_{0,4 k}, x_{0}, x_{0,1}, x_{0,1,1}, \ldots, x_{0,1,1, \ldots, 1}, \ldots
$$

If $x \notin \gamma$, it is labelled as $x_{0, i_{1}, i_{2}, \ldots, i_{l}}$, where either $i_{1} \neq 4 k$ and some $i_{j} \neq 1$ for some $j \geq 1$ or $i_{1}=4 k$ and $i_{j} \neq 1$ for some $j \geq 2$.

If $i_{1} \neq 4 k$ let $j_{0}=\min \left\{j=1,2,3, \ldots, l \mid i_{j} \neq 1\right\}$ be the first index in its label different from 1 and put

$$
\phi\left(x_{0, i_{1}, 1, \ldots, 1, i_{j_{0}}, \ldots, i_{l}}\right):=x_{0, i_{1}, 1, \ldots, 1, \sigma\left(i_{j_{0}}\right), \ldots, i_{l}} .
$$


If $i_{1}=4 k$ let $j_{0}=\min \left\{j=2,3, \ldots, l \mid i_{j} \neq 1\right\}$ be the index of the first label different from 1 (except the first entry) and put $\phi\left(x_{\left.0, i_{1}, 1, \ldots, 1, i_{j_{0}}, \ldots, i_{l}\right)}\right):=x_{0, i_{1}, 1, \ldots, 1, \sigma\left(i_{j_{0}}\right), \ldots, i_{l}}$.

From its very construction, the restriction of $\phi$ to $B\left(x_{0}, R\right)$ coincides with $\varphi$. Moreover, the only fixed points of $\phi$ are the ones in the geodesic labeled as

$$
\ldots, x_{0,4 k, 1, \ldots, 1}, \ldots, x_{0,4 k, 1}, x_{0,4 k}, x_{0}, x_{0,1}, x_{0,1,1}, \ldots, x_{0,1,1, \ldots, 1}, \ldots
$$

and

$$
\begin{aligned}
\phi^{2}\left(x_{0, i_{1}, 1, \ldots, 1, i_{j_{0}}, \ldots, i_{l}}\right) & =\phi\left(x_{0, i_{1}, 1, \ldots, 1, \sigma\left(i_{j_{0}}\right), \ldots, i_{l}}\right) \\
& =x_{0, i_{1}, 1, \ldots, 1, \sigma\left(\sigma\left(i_{j_{0}}\right)\right), \ldots, i_{l}} \\
& =x_{0, i_{1}, 1, \ldots, 1, i_{j_{0}}, \ldots, i_{l}}
\end{aligned}
$$

and it follows that $\phi$ is an involution fixing exactly one geodesic, that is, $\phi$ is a reflection whose restriction to $B$ is $\varphi$.

We want now to prove that, given an isometry $\psi: \Gamma \rightarrow \Gamma$ that fixes a vertex of $\Gamma$, its action on the vertices adjacent to the given fixed point may be produced by reflections (Proposition 9).

In the next lemma, we will consider isometries $\psi_{i j}$ that fixes $x_{0}$ and all of its adjacent vertices, except for two of them (labeled by the indices $i$ and $j$ ), that is, elements that restricted to $S\left(x_{0}, 1\right)$ acts as transpositions.

Lemma 8 Let $\Gamma$ be a homogeneous tree with Degree $(\Gamma)=4 k$. Let $x_{0} \in \Gamma$ and $x_{0,1}, x_{0,2}, \ldots, x_{0,4 k}$ be the $4 k$-vertices of $\Gamma$ adjacent to $x_{0}$. For a given pair of indices $i, j$, let $\psi_{i j}$ be an isometry such that

$$
\begin{aligned}
\psi_{i j}\left(x_{0}\right) & =x_{0}, \\
\psi_{i j}\left(x_{0, i}\right) & =x_{0, j}, \\
\psi_{i j}\left(x_{0, j}\right) & =x_{0, i}, \\
\psi_{i j}\left(x_{0, n}\right) & =x_{0, n} \text { for } n \neq i, j .
\end{aligned}
$$

Then there are reflections $\phi_{1}, \phi_{2}, \phi_{3}$ fixing the vertex $x_{0}$, such that

$$
\left.\psi_{i j}\right|_{B\left(x_{0}, 1\right)}=\left.\phi_{1} \circ \phi_{2} \circ \phi_{3}\right|_{B\left(x_{0}, 1\right)} .
$$

Proof. We consider the restriction of $\psi_{i j}$ to the set

$$
S\left(x_{0}, 1\right):=\left\{x_{0,1}, \ldots, x_{0,4 k}\right\}
$$

of vertices adjacent to $x_{0}$. We may rename those vertices in such a way that $x_{0, i}=x_{0,1}$ and $x_{0, j}=x_{0,2}$. Consider the permutations $\sigma_{1}, \sigma_{2}, \sigma_{3} \in S_{4 k}$ defined as

$$
\begin{aligned}
& \sigma_{1}=(1,2)(5,6)(7,8) \cdots(4 k-3,4 k-2)(4 k-1,4 k) \\
& \sigma_{2}=(3,4)(5,7)(6,8) \cdots(4 k-3,4 k-1)(4 k-2,4 k) \\
& \sigma_{3}=(3,4)(5,8)(6,7) \cdots(4 k-3,4 k)(4 k-2,4 k-1) .
\end{aligned}
$$


Direct computation shows that $\sigma_{1} \circ \sigma_{2} \circ \sigma_{3}=(1,2)$. For $i=1,2,3$ we define $\bar{\phi}_{i} \in \mathcal{R}_{B,\left[x_{0,1}, x_{0,2}\right]}$ as

$$
\begin{aligned}
\bar{\phi}_{i}\left(x_{0}\right) & =x_{0} \\
\bar{\phi}_{i}\left(x_{0, l}\right) & =x_{0, \sigma_{i}(l)} .
\end{aligned}
$$

The previous proposition assures that each $\bar{\phi}_{i}$ can be extended to a reflection $\phi_{i}$ of $\Gamma$. But

$$
\begin{aligned}
\phi_{1} \circ \phi_{2} \circ \phi_{3}\left(x_{0}\right) & =\bar{\phi}_{1} \circ \bar{\phi}_{2} \circ \bar{\phi}_{3}\left(x_{0}\right) \\
& =x_{0} \\
& =\psi_{i j}\left(x_{0}\right)
\end{aligned}
$$

and for $x_{0, l} \in S\left(x_{0}, 1\right)$ we find that

$$
\begin{aligned}
\phi_{1} \circ \phi_{2} \circ \phi_{3}\left(x_{0, l}\right) & =\bar{\phi}_{1} \circ \bar{\phi}_{2} \circ \bar{\phi}_{3}\left(x_{0, l}\right) \\
& =x_{0, \sigma_{1} \circ \sigma_{2} \circ \sigma_{3}(l)} \\
& =\psi_{i j}\left(x_{0, l}\right)
\end{aligned}
$$

so that

$$
\left.\psi_{i j}\right|_{B\left(x_{0}, 1\right)}=\left.\phi_{1} \circ \phi_{2} \circ \phi_{3}\right|_{B\left(x_{0}, 1\right)} .
$$

Proposition 9 In the same conditions as in the previous lemma, given an isometry $\psi: \Gamma \longrightarrow \Gamma$, such that $\psi\left(x_{0}\right)=x_{0}$, there are reflections $\phi_{1}, \phi_{2}, \ldots, \phi_{l}$ fixing the vertex $x_{0}$, such that $\phi_{1} \circ \phi_{2} \circ \ldots \circ \phi_{l}\left(x_{0, n}\right)=\psi\left(x_{0, n}\right)$, for every $n \in\{1,2, \ldots, 4 k\}$.

Proof. Since $\psi$ fixes the vertex $x_{0}$, its restriction acts as a permutation of $S\left(x_{0}, 1\right)=$ $\left\{x_{0,1}, x_{0,2}, \ldots, x_{0,4 k}\right\}$. Since any permutation may be expressed as a product of transpositions and, by the previous lemma, any transposition of $S\left(x_{0}, 1\right)$ may be produced by reflections, the restriction of $\psi$ to $B\left(x_{0}, 1\right)$ may be produced by reflections.

We remark that both in the proposition as in the lemma that precedes it, the hypothesis that Degree $(\Gamma) \equiv 0 \bmod 4$ is essential. Indeed, given a reflection $\phi$ that fixes a point $x_{0}$, its restriction to the vertices adjacent to $x_{0}$ is expressed as a product

$$
\left(x_{0, j_{1}} x_{0, j_{2}}\right)\left(x_{0, j_{3}} x_{0, j_{4}}\right) \cdots\left(x_{0, j_{N-3}} x_{0, j_{N-2}}\right),
$$

of disjoint transpositions, where $N=\operatorname{Degree}(\Gamma)$. If $N=4 k+2$, the reflection $\phi$ involves an even $(2 k)$ number of transpositions. Hence, any product of reflections fixing $x_{0}$, when restricted to the sphere $S\left(x_{0}, 1\right)$ corresponds to an element of the alternating group. It follows that an isometry that fixes $x_{0}$ and all but two of the adjacent vertices may not be expressed as a product of reflections fixing $x_{0}$.

The next lemma is the first step needed to extend the proposition 9, passing from the sphere $S\left(x_{0}, n\right)$ to $S\left(x_{0}, n+1\right)$. 
Lemma 10 Given distinct vertices $x_{0}, v_{1}, v_{2}$ of a homogeneous tree $\Gamma$, with Degree $(\Gamma)=$ $4 k$, such that $d\left(v_{1}, x_{0}\right)=d\left(v_{2}, x_{0}\right)=R$, let $w_{1}^{1}, w_{2}^{1}, w_{1}^{2}, w_{2}^{2} \in B\left(x_{0}, R+1\right)$ be distinct vertices such that $w_{j}^{i}$ is adjacent to $v_{i}$, for $i, j=1,2$. Then, there are reflections $\phi$ and $\psi$ such that:

1. $\phi\left(x_{0}\right)=\psi\left(x_{0}\right)=x_{0}$;

2. $\phi \circ \psi\left(w_{1}^{i}\right)=w_{2}^{i}$ and $\phi \circ \psi\left(w_{2}^{i}\right)=w_{1}^{i}$, for $i=1,2$;

3. $\phi \circ \psi(x)=x$ if $d\left(x, x_{0}\right) \leq R+1$ and $x \neq w_{j}^{i}$, for $i, j=1,2$.

Proof. Since $d\left(v_{1}, x_{0}\right)=d\left(v_{2}, x_{0}\right)=R$, it follows that $d\left(v_{1}, v_{2}\right)$ is even. Let $x_{1}$ be the middle point of the geodesic segment $\left[v_{1}, v_{2}\right]$. We let $\gamma$ be a geodesic containing the segment $\left[x_{0}, x_{1}\right]$ such that $\gamma \cap\left[v_{1}, v_{2}\right]=\left\{x_{1}\right\}$. It follows that for every $N \geq 0, \gamma \cap B\left(x_{0}, N\right)$ is a diameter $\left[a_{N}, b_{N}\right]$ of $B\left(x_{0}, N\right)$. We let $\bar{\varphi} \in \mathcal{R}_{B,\left[a_{R}, b_{R}\right]}$ be any reflection of $B\left(x_{0}, R\right)$ such that $\bar{\varphi}\left(v_{1}\right)=v_{2}$ and (consequently) $\bar{\varphi}\left(v_{2}\right)=v_{1}$. The existence of $\bar{\varphi}$ is easily established, e.g. by the method used in the proof of Proposition 7 .

We label the vertices of $S\left(x_{0}, R\right)$ as $\left\{v_{1}, v_{2}, \ldots, v_{l}\right\}$. We note that, since Degree $(\Gamma)=$ $4 k$, we have $l=4 k(4 k-1)^{R-1}$. Each $v_{i}$ has exactly $4 k-1$ adjacent vertices at distance $R+1$ from $x_{0}$. We label them as $w_{1}^{i}, w_{2}^{i}, \ldots, w_{4 k-1}^{i}$. We let $\varphi \in S_{l}$ be such that $\bar{\varphi}\left(v_{i}\right)=v_{\varphi(i)}$ for every $i \in\{1,2, \ldots, l\}$. Since $\bar{\varphi}$ is a reflection in a diameter of $B\left(x_{0}, R\right)$, its restriction to $S\left(x_{0}, R\right)$ is a product of $\frac{l-2}{2}$ disjoint transpositions. We may assume $\varphi=(1,2) \cdots(l-3, l-2)$. With this notation we find that $\left[a_{R}, b_{R}\right]=\left[v_{l-1}, v_{l}\right]$.

We will define permutations $\bar{\phi}$ and $\bar{\psi}$ of $B\left(x_{0}, R+1\right)$ to coincide with $\bar{\varphi}$ when restricted to $B\left(x_{0}, R\right)$. To extend those permutations to $S\left(x_{0}, R+1\right)$ we consider $\alpha \in S_{4 k-1}$ defined as the product of disjoint transpositions

$$
(2,3)(4,5) \cdots(4 k-2,4 k-1)
$$

and proceed in the following way:

$$
\begin{aligned}
& \bar{\phi}\left(w_{1}^{1}\right):=w_{2}^{2} ; \bar{\phi}\left(w_{2}^{2}\right)=w_{1}^{1} \\
& \bar{\phi}\left(w_{2}^{1}\right):=w_{1}^{2} ; \bar{\phi}\left(w_{1}^{2}\right)=w_{2}^{1} \\
& \bar{\phi}\left(w_{j}^{i}\right):=w_{\alpha(j)}^{\varphi(i)} \text { for }\{i, j\} \nsubseteq\{1,2\} ; \\
& \bar{\psi}\left(w_{1}^{1}\right):=w_{1}^{2} ; \bar{\psi}\left(w_{2}^{2}\right)=w_{2}^{1} \\
& \bar{\psi}\left(w_{2}^{1}\right):=w_{2}^{2} ; \bar{\psi}\left(w_{1}^{2}\right)=w_{1}^{1} \\
& \bar{\psi}\left(w_{j}^{i}\right):=w_{\alpha(j)}^{\varphi(i)} \text { for }\{i, j\} \nsupseteq\{1,2\} .
\end{aligned}
$$

Recalling that a permutation of vertices is an automorphism of a graph if and only if it preserves adjacency, we see that it is easy to show that $\bar{\phi}$ and $\bar{\psi}$ are automorphism of $B\left(x_{0}, R+1\right)$. Since both $\varphi$ and $\alpha$ are involutions, we find that $\bar{\phi}^{2}=\bar{\psi}^{2}=\left.\operatorname{Id}\right|_{B\left(x_{0}, R+1\right)}$. Moreover, the set of fixed points of $\bar{\phi}$ and $\bar{\psi}$ is exactly the diameter $\left[v_{l-1}, v_{l}\right]$ (in $B\left(x_{0}, R\right)$ ) 
and the additional vertices $w_{1}^{l-1}$ and $w_{1}^{l}$, adjacent to $v_{l-1}$ and $v_{l}$ respectively. In resume, $\bar{\phi}$ and $\bar{\psi}$ are automorphisms of order 2 of $B\left(x_{0}, R+1\right)$ that have the diameter $\left[a_{R+1}, b_{R+1}\right] \subset$ $\gamma$ as set of fixed points. In other words, $\bar{\phi}, \bar{\psi} \in \mathcal{R}_{B\left(x_{0}, R+1\right),\left[a_{R+1}, b_{R+1}\right]}$. Proposition 7 assures we can extend $\bar{\phi}$ and $\bar{\psi}$ to reflections $\phi$ and $\psi$ of $\Gamma$.

Since $\left.\bar{\phi}\right|_{B\left(x_{0}, R\right)}=\left.\bar{\psi}\right|_{B\left(x_{0}, R\right)}=\bar{\varphi}$, and $\bar{\varphi}$ is a reflection, we find that $\left.\bar{\phi} \circ \bar{\psi}\right|_{B\left(x_{0}, R\right)}$ is the identity. Direct calculation shows that

$$
\begin{aligned}
\left.\bar{\phi} \circ \bar{\psi}\right|_{S\left(x_{0}, R+1\right)} & =\left(w_{1}^{1} w_{2}^{2}\right)\left(w_{2}^{1} w_{1}^{2}\right)\left(w_{1}^{1} w_{1}^{2}\right)\left(w_{2}^{1} w_{2}^{2}\right) \\
& =\left(w_{1}^{1} w_{2}^{1}\right)\left(w_{2}^{21} w_{1}^{2}\right)
\end{aligned}
$$

and it means the reflections $\phi$ and $\psi$ of $\Gamma$ satisfy the required conditions.

The preceding lemma assures that we can transpose vertices in a sphere, maintaining all other vertices in the closed metric ball fixed. However, those transpositions are done simultaneously in two pairs of distinct vertices. In order to extend Proposition 9 from a ball of radius $R$ to a ball of radius $R+1$, we need to transpose only one chosen pair of vertices. This is done in the following lemma, by considering a sufficiently large ball, that contains the given ball of radius $R$.

Lemma 11 In the same conditions as before, let $v, x_{0}$ be vertices of $\Gamma$ such that $d\left(v, x_{0}\right)=R$. Let $w_{1}, w_{2} \in B\left(x_{0}, R+1\right)$ be vertices adjacent to $v$. Then, there are reflections $\phi$ and $\psi$ such that:

1. $\phi \circ \psi\left(w_{1}\right)=w_{2}$ and $\phi \circ \psi\left(w_{2}\right)=w_{1}$;

2. $\phi \circ \psi(x)=x$ if $d\left(x, x_{0}\right) \leq R+1$ and $x \neq w_{i}$, for $i=1,2$.

Proof. Let $y_{0}$ be a vertex of $\Gamma$ such that

$$
\begin{aligned}
& d\left(y_{0}, x_{0}\right)=1 \\
& d\left(v, y_{0}\right)>d\left(v, x_{0}\right)
\end{aligned}
$$

Denote $v=v_{1}, w_{1}=w_{1}^{1}, w_{2}=w_{2}^{1}$ and let $v_{2}$ be a vertex such that $y_{0}$ is the middle point of the geodesic segment $\left[v_{1}, v_{2}\right]$. We choose now $w_{1}^{2}$ and $w_{2}^{2}$ satisfying

$$
\begin{aligned}
& d\left(w_{1}^{2}, v_{2}\right)=d\left(w_{2}^{2}, v_{2}\right)=1 \\
& d\left(w_{i}^{2}, y_{0}\right)>d\left(v_{2}, y_{0}\right), \text { for } i=1,2 .
\end{aligned}
$$

By the previous lemma, there are reflections $\phi$ and $\psi$ such that

$$
\begin{aligned}
\phi\left(y_{0}\right) & =\psi\left(y_{0}\right)=y_{0} \\
\phi \circ \psi\left(w_{1}^{i}\right) & =w_{2}^{i}, \quad \phi \circ \psi\left(w_{2}^{i}\right)=w_{1}^{i} \text { for } i=1,2 \\
\phi \circ \psi(x) & =x \text { if } d\left(x, y_{0}\right) \leq R+2 \text { and } x \neq w_{j}^{i}, i, j=1,2 .
\end{aligned}
$$

By construction, we find that $B\left(x_{0}, R+1\right) \subset B\left(y_{0}, R+2\right)$ and also

$$
d\left(w_{i}^{2}, x_{0}\right)=d\left(w_{i}^{2}, y_{0}\right)+d\left(y_{0}, x_{0}\right)=R+3 .
$$


It follows that

$$
\begin{aligned}
\phi \circ \psi\left(w_{1}\right) & =w_{2}, \phi \circ \psi\left(w_{2}\right)=w_{1} \\
\phi \circ \psi(x) & =x \text { if } d\left(x, x_{0}\right) \leq R+1 \text { and } x \neq w_{i}, \text { for } i=1,2 .
\end{aligned}
$$

We are able now to make the inductive step:

Proposition 12 Let $\Gamma$ be a homogeneous tree with Degree $(\Gamma) \equiv 0 \bmod 4$. Let $x_{0}$ be a vertex of $\Gamma$ and $\varphi: \Gamma \longrightarrow \Gamma$ an isometry such that $\varphi\left(x_{0}\right)=x_{0}$. Then, for any $R \geq 1$, there are reflections $\phi_{1}, \phi_{2}, \ldots, \phi_{l}$ such that $\left.\varphi\right|_{B\left(x_{0}, R\right)}=\left.\phi_{1} \circ \phi_{2} \circ \ldots \circ \phi_{l}\right|_{B\left(x_{0}, R\right)}$.

Proof. We prove by induction on $R$.

The case $R=1$ was proved in Proposition 9 .

Assuming it holds for $R-1$, there are reflections $\phi_{1}, \phi_{2}, \ldots, \phi_{m}$ such that

$$
\left.\varphi\right|_{B\left(x_{0}, R-1\right)}=\left.\phi_{1} \circ \phi_{2} \circ \ldots \circ \phi_{m}\right|_{B\left(x_{0}, R-1\right)} .
$$

Let $v$ be a vertex with $d\left(v, x_{0}\right)=R-1$ and let $w_{1}, w_{2}, \ldots, w_{4 k-1}$ be the vertices adjacent to $v$ with $d\left(w_{i}, x_{0}\right)=R$, for $i=1,2, \ldots, 4 k-1$. Let $v^{\prime}=\varphi(v)$ and $w_{i}^{\prime}=\varphi\left(w_{i}\right)$. Since $d\left(v, x_{0}\right)=R-1$, we have that

$$
v^{\prime}=\phi_{1} \circ \phi_{2} \circ \ldots \circ \phi_{m}(v)
$$

Moreover,

$$
\phi_{1} \circ \phi_{2} \circ \ldots \circ \phi_{m}\left(w_{i}\right)=w_{j}^{\prime} \text { for some } j \in\{1,2, \ldots, 4 k-1\} .
$$

To conclude the proof, we just note again that any permutation may be produced by transpositions and then apply the previous lemma.

We consider on Aut $(\Gamma)$ the topology of uniform convergence over compact sets. Since $\Gamma$ is discrete, this is equivalent to say that a sequence $\phi_{n} \in \operatorname{Aut}(\Gamma)$ converges to $\phi_{0}$ if and only if, for any finite subset $K$ of vertices of $\Gamma$, there is an $N \geq 0$ such that, for every $x \in K, \phi_{n}(x)=\phi_{0}(x)$ if $n \geq N$. With this topology, we find that, for a given subgroup $G \subset \operatorname{Aut}(\Gamma)$, its topological closure $\bar{G}$ is a subgroup of Aut $(\Gamma)$. If $G=\langle\mathcal{R}\rangle$ is generated by a set $\mathcal{R}$, a typical element $\phi \in \overline{\langle\mathcal{R}\rangle}$ is given by a sequence $\left(\phi_{n}\right)_{n \in \mathbb{N}}$ of elements of $\mathcal{R}$ such that

$$
\phi(x)=\lim _{n \longrightarrow \infty} \phi_{n} \circ \phi_{n-1} \circ \ldots \circ \phi_{1}(x)
$$

exist for every vertex $x \in \Gamma$ and such that $\phi(x) \in \operatorname{Aut}(\Gamma)$. The sequence may be taken as finite if $\phi \in G$.

Remark 13 As privately pointed by Gadi Moran, every reflection $\phi \in \mathcal{R}_{x_{0}}$ has the property that its restriction to every sphere $S\left(x_{0}, N\right)$ is an odd permutation. It follows that for any finite sequence $\phi_{1}, \ldots, \phi_{n} \in \mathcal{R}_{x_{0}}$, the restriction of the product $\phi_{1} \circ \phi_{2} \circ \cdots \circ \phi_{n}$ to every sphere $S\left(x_{0}, N\right)$ has the same parity as the index $n$. The subgroup $H \subset$ Aut $(\Gamma)_{x_{0}}$ of all 
automorphisms with this property, namely having the same parity when restricted to any given sphere $S\left(x_{0}, N\right)$, is a closed and proper subgroup of Aut $(\Gamma)_{x_{0}}$. It follows that the closure of $\left\langle\mathcal{R}_{x_{0}}\right\rangle$ is also a proper subgroup of Aut $(\Gamma)_{x_{0}}$. In Lemma 11, we have a pair of reflections whose composition $\phi \circ \psi$ is the identity when restricted to $B\left(x_{0}, R\right)$, and hence an even permutation on every $S\left(x_{0}, N\right), N \leq R$. However, restricted to $S\left(x_{0}, R+1\right)$ it is an odd permutation, defined by a single transposition. The contradiction is only an appearance, since the common fixed point of the reflections $\phi$ and $\psi$ is not $x_{0}$, the center of the spheres in consideration.

With those definitions and terminology, the previous proposition can be restated as follows:

Proposition 14 Let $\Gamma$ be a homogeneous tree with Degree $(\Gamma) \equiv 0 \bmod 4 . \quad$ Let $\mathcal{R}=\{\phi \in A$ ut $(\Gamma) \mid \phi$ is a reflection $\}$. Given $x_{0} \in \Gamma$, denote by Aut $(\Gamma)_{x_{0}}$ the stabilizer of $x_{0}$ in Aut $(\Gamma)$. Then Aut $(\Gamma)_{x_{0}} \subseteq \overline{\langle\mathcal{R}\rangle}$.

Proof. It follows from proposition 12 , since any finite set of vertices is contained in ball $B\left(x_{0}, R\right)$, for $R$ sufficiently large.

We recall that $\mathrm{Aut}^{+}(\Gamma)$ is the set of automorphisms $\phi$ with even displacement function $d_{\phi}$.

Theorem 15 Let $\Gamma$ be a homogeneous tree with Degree $(\Gamma) \equiv 0 \bmod 4$. Let $\mathcal{R}$ be the set of reflections of $\Gamma$. Then the closure $\overline{\langle\mathcal{R}\rangle}$ of the group generated by reflections is the group $\operatorname{Aut}^{+}(\Gamma)$, a normal subgroup of index 2 in Aut $(\Gamma)$.

Proof. Since every reflection has even displacement function (Lemma 6), $\overline{\langle\mathcal{R}\rangle} \subseteq \mathrm{Aut}^{+}(\Gamma)$. We shall prove the other inclusion.

If $\phi \in \operatorname{Aut}^{+}(\Gamma)$ has a fixed point $x_{0}$, then $\phi \in \operatorname{Aut}(\Gamma)_{x_{0}} \subseteq \overline{\langle\mathcal{R}\rangle}$. So, we assume that $\phi$ has no fixed points. Since $d_{\phi}(x) \equiv 0 \bmod 2$ for any vertex $x$, there is a middle point $p:=p(x)$ of the segment $[x, \phi(x)]$. With the same methods used in the proof of Proposition 7, we can prove there is a reflection $\varphi \in \mathcal{R}_{p}$ such that $\varphi(\phi(x))=x$. It follows that $\varphi \circ \phi \in \overline{\langle\mathcal{R}\rangle}$, since it has $x$ as a fixed point. But $\varphi$ also belongs to $\overline{\langle\mathcal{R}\rangle}$, and this implies that $\phi=\varphi \circ \varphi \circ \phi \in \overline{\langle\mathcal{R}\rangle}$, and we find that $\operatorname{Aut}^{+}(\Gamma) \subseteq \overline{\langle\mathcal{R}\rangle}$.

Acknowledgement: We acknowledge Gadi Moran for the patience to read and correct this paper too many times, and also for the many suggestions and remarks that enriched this work.

\section{References}

[1] I. Chiswell., Introduction to $\Lambda$-Trees (World Scientific Publishing, 2001).

[2] G. Moran, 'Trees and the bireflection property', Israel Journal of Mathematics 41 vol. 3 (1982), 244-260.

[3] R. Möller, 'The automorphism groups of regular trees', J. London Math. Soc. 43, vol. 2 (1991), 236-252.

[4] J. P. Serre, Trees (Springer Verlag, 1980). 